\title{
Aufbruch, Brüche und Entfaltung im Auseinanderbrechen: Historischer Abriss der brasilianischen Psychoanalyse
}

Gregor Busslinger (Zürich)

Zusammenfassung: Der Ausgangspunkt ist eine aktuelle Erfahrung mit einem psychoanalytischen Institut in Rio de Janeiro, das mich mit einem schwierigen Kapitel europäischer Geschichte konfrontierte. Danach gehe ich den Entstehungsbedingungen der Psychoanalyse in Brasilien nach und zeige auf, wie die heutige Praxis dadurch beeinflusst ist. So war der Motor für die Entwicklung der Psychoanalyse in den zwei Zentren São Paulo und Rio de Janeiro recht unterschiedlich, was spannende Fragen aufwirft. In der weiteren Entwicklung der psychoanalytischen Bewegung im Kontext der Diktaturen der 60er- und 70erJahre haben sich daraus Konflikte ergeben, in denen es um die Situierung der Psychoanalyse bezüglich berufspolitischer, sozialer und politischer Fragen ging. Daraus entwickelte sich eine facettenreiche psychoanalytische Landschaft.

Schlüsselwörter: Institutionalisierung, Geschichte der brasilianischen Psychoanalyse, Spaltungsgeschichte, Institutionskritik, Modernismus

\section{Annäherung auf Um- und Abwegen}

Der Ausgangspunkt meiner aktuellen Auseinandersetzung mit der brasilianischen Psychoanalyse ist ein Vortrag zur Ethnopsychoanalyse, den ich im April 2014 im Círculo de Psicanálise de Rio de Janeiro (CPRJ) hielt. Der CPRJ ist ein psychoanalytisches Institut, das bezüglich seiner Organisation und seiner Situierung in der psychoanalytischen Landschaft Ähnlichkeiten mit dem Psychoanalytischen Seminar Zürich (PSZ) aufweist. Die verschiedenen psychoanalytischen Schulen (Freud, Klein, Winnicott, Bion, Lacan) werden unter einem Dach vermittelt. Der Zugang ist für die verschiedensten universitären Studiengänge offen und die Organisationsform ist demokratisch (siehe: http://www.cprj.com.br). Von meiner bisherigen Auseinandersetzung mit der brasilianischen Psychoanalyse, die in einem Journalartikel 2004 ihren Niederschlag fand, wusste ich, dass die Ursprünge des CPRJ auf Ana Katrin Kemper zurückgehen. Diese vertrat in den 
60er-Jahren eine kritische Haltung gegenüber der Abschottung und der Hierarchie der International Psychoanalytic Association (IPA)-Gesellschaften. Da ihr psychoanalytischerWerdegang aber unter diffusen Umständen im Umkreis des GöhringInstituts begann, löste ihrWirken in Brasilien - aber auch in der Zeitschrift Psychediverse Kontroversen aus (Schneider, 1985). Neu für mich war dagegen, dass bei der Gründung des CPRJ 1969 Igor Caruso Pate stand. Neugierig begann ich während meines Aufenthaltes in Rio de Janeiro, online über Caruso zu recherchieren, da er mir als Vertreter einer progressiven Psychoanalyse ein diffuser Begriff war. Caruso gründete 1947 in Abgrenzung gegenüber dem Medicozentrismus der Wiener Psychoanalytischen Vereinigung (WPV) den Wiener Arbeitskreis für Tiefenpsychologie (WATP). Ab 1956 war er massgeblich bei der Gründung diverser psychoanalytischer Arbeitskreise in Lateinamerika beteiligt, neben Brasilien auch in Argentinien, Mexiko und Kolumbien. Verblüfft stellte ich fest, dass ich bei Wikipedia je nach verwendeter Sprache auf ziemlich unterschiedliche Informationen stiess. In Portugiesisch fand ich kurze Einträge zu seinem progressiven Wirken in der psychoanalytischen Landschaft Lateinamerikas, währenddessen man in Deutsch seinen Werdegang ausführlicher nachlesen kann. Unter anderem ist zu lesen:

\begin{abstract}
Caruso war von Februar 1942 bis Oktober 1942 unter der Leitung von Ernst Illing und dem Stationsarzt Heinrich Gross Erzieher und psychologischer Gutachter in der «Kinderfachabteilung», den Pavillons 15 und 17 (Abteilungen "Ausmerzende Maßnahmen» und «Erb- und Rassenpflege») der Wiener «Fürsorgeanstalt» Spiegelgrund. Mindestens 14 Kinder wurden auch aufgrund der von ihm erstellten psychologischen Gutachten im Zuge des KinderEuthanasie-Programmes ermordet. (http:// de.wikipedia.org/wiki/ Igor_Alexander_Caruso)
\end{abstract}

Bestürzt berichtete ich davon dem Mitglied der Institutsleitung des CPRJ, das mich zum Vortrag eingeladen hatte. Sie konnte es kaum glauben, meinte dann resigniert, dass ein solcher Sachverhalt kaum thematisierbar sei, solange Jochen Kemper, der Sohn der Begründerin des CPRJ, ebenfalls ein wichtiges Mitglied des Instituts, der seine Mutter in einem Psyche-Artikel in den 80er-Jahren vehement verteidigt hatte (Kemper, 1988), noch am Leben sei. Zurück in der Schweiz recherchierte ich über den «Fall Caruso» - und stiess auf ein Stück schwierige Geschichte der Psychoanalyse im deutschen Sprachraum. Obwohl Caruso 1979 in einer Radiosendung im österreichischen Rundfunk durchaus nicht beschö- 
nigend von seiner Verwicklung in das Nazi-Euthanasie-Programm erzählt hatte, wurde er als eine der Ikonen der 68er-Bewegung, der seit 1967 einer Professur für Klinische Psychologie und Sozialpsychologie in Salzburg innehatte, mit keinem Wort erwähnt, als das SalzburgerWerkblatt in den 80er-Jahren eine Debatte über die Verwicklung der Psychoanalyse mit dem Nationalsozialismus führte. Karl Fallend schreibt dazu im Werkblatt mehr als 20 Jahre später:

Wir hörten Vorträge und diskutierten über Waldheim, die Vertreibung der Psychoanalyse, über August Aichhorn im nationalsozialistischen Wien, die apolitisch institutionalisierte Psychoanalyse im "Dritten Reich», gar über die Geschichte der Euthanasie und selbst Werner Vogt hatten wir eingeladen, um u.a. über Heinrich Gross und den organisierten Mord am Spiegelgrund zu referieren. Über Igor Caruso - zumindest meiner Erinnerung nach - kein Wort. (Fallend, 2010, S. 111)

Erst zur Zeit dieser Feststellung von Fallend fand im Werkblatt die längst überfällige schwierige Auseinandersetzung mit einer der wichtigen Identifikationsfiguren der progressiven Psychoanalyse statt (Werkblatt Nr. 62-65). Mit diesen Ausführungen hege ich keinen Anspruch, dem Werdegang von Igor Caruso und der Debatte über ihn gerecht zu werden. Aber der in groben Zügen skizzierte Umgang mit dem «Fall Caruso» in unseren Breitengraden macht die Aussage meiner Gastgeberin in Rio zur Schwierigkeit der Vergangenheitsbewältigung mehr als verständlich. In der Geschichte der Psychoanalyse mangelt es nicht an Zeugnissen dieser Schwierigkeit. Wenn ich im Folgenden der Entwicklung der brasilianischen Psychoanalyse nachgehe, werde ich diese schwierige Vergangenheitsbewältigung nur am Rande streifen. Auf die Komplizenschaft der offiziellen brasilianischen Psychoanalyse und der IPA mit der repressiven Herrschaft der Militärs zwischen 1964 und 1985 bin ich im Aufsatz «Die États Généraux de la Psychanalyse in Rio de Janeiro und die brasilianische Psychoanalyse» (Busslinger, 2004) ausführlich eingegangen.

\section{Aufbruch}

Die brasilianische Psychoanalyse entwickelte sich in den Anfängen auf je unterschiedlichen Wegen zuerst in den zwei grossen Zentren Rio de Janeiro und São Paulo. Eingeführt wurde das psychoanalytische Gedankengut aber weitab von diesen Zentren, von Juliano Moreira, der eine Sozialisation als Angehöriger 
der afrobrasilianischen Unterschicht im Nordosten Brasiliens durchlief. Als junger Psychiatrieprofessor dozierte er an der Universität in Salvador da Bahia 1899, noch vor der Publikation der Traumdeutung, freudsches Gedankengut. Ab 1903 leitete er das “Hospício Nacional de Alienados”, das «staatliche Krankenhaus für Geistesgestörte» in Rio de Janeiro, zu einer Zeit als der herrschende Diskurs unter den Medizinern in der Rassenvermischung des brasilianischen Volkes eine grosse Gefahr für die "higiene mental” (Psychohygiene) sah. Verhaftet im Zeitgeist stellte er zwar die herrschende Ideologie der geistigen Degeneration nie in Frage, kämpfte hingegen dafür, dass die Ursachen nicht mehr an der Rassenfrage sondern an den schwierigen sozialen und hygienischen Bedingungen festgemacht wurden (Galdini, 2001). Als Anhänger von Emil Kraepelin spielte Moreira keine aktive Rolle bei der Auseinandersetzung mit der psychoanalytischen Theorie. Er produzierte nie psychoanalytische Texte, sondern nahm lediglich an derVerbreitung derselben teil. Als einflussreiche Figur in den medizinischen Kreisen war er aber ähnlich wie Eugen Bleuler bei uns einer ihrer Wegbereiter. Während seiner Leitung des "Hospício Nacional de Alienados" zwischen 1903 und 1930 formierte sich dort die "guardia psicanalítica” (psychoanalytische Avantgarde) (Plotkin, 2009, S. 67). Als Begründer der modernen brasilianischen Psychiatrie (Roudinesco \& Plon, 2004) war er auch, wie Bleuler, massgeblich an der Psychiatriereform des beginnenden 20. Jahrhunderts beteiligt und ebenfalls wie dieser ganz im Sinne des Zeitgeistes ein Verfechter der Eugenik. In Rio de Janeiro, wo er wirkte, entwickelte sich die Psychoanalyse im Schoss der Ideologie der "higiene mental”, in welcher ein wichtiger Antrieb für den Fortschritt des brasilianischen Volkes gesehen wurde.

Obwohl die Psychoanalyse anfänglich in Rio aufkam, gewann sie zuerst über die Verbreitung in São Paulo an Einfluss. Franco de Rocha, der Begründer der psychiatrischen Klinik Juquery in São Paulo, dozierte als Professor an der medizinischen Fakultät psychoanalytisches Gedankengut und veröffentlichte 1920 das Buch "O Pansexualismo na Doutrina de Freud" (Pansexualität in der Lehre Freuds), in dem er Freuds These der «Drei Abhandlungen zur Sexualtheorie» von 1905 vertrat. Seine Thesen lösten heftige Diskussionen und Kritik an der medizinischen Fakultät aus und führten zu Besorgnis um den Gesundheitszustand des Autors (Mokrejs, 1992). In literarischen Kreisen dagegen stiess seine Initiative auf grosses Interesse. In ihrem Buch «Tupi Talking Cure. Zur Aneignung Freuds im antropofagischen Modernismus Brasiliens» beschreibt Catrin Seefranz ausführlich, wie die Modernisten anlässlich der "Semana de Arte Moderna” 1922 in São Paulo - einem kulturellen Ereignis der Avantgarde mit grosser Ausstrahlung - die Thesen des «Pansexualismus» aufnahmen. Die antropofagische Bewegung der 
brasilianischen Modernisten zielte darauf ab, sich in antikolonialem Geist die kulturellen, ästhetischen und politischen Diskurse aus Europa und Nordamerika einzuverleiben, das Verwertbare aufzunehmen und den Rest auszustossen, um etwas Eigenes daraus zu machen und dadurch ein neues Selbstbewusstsein zu entwickeln. Die metaphorische Bedeutung der Menschenfresserei bezog sich aber nicht nur auf die Aneignung und Einverleibung des neuen Anderen, sondern auch auf eine Rückbesinnung auf die eigenen Wurzeln, auf die vorkolonialen Zustände, auf die Tupis, welche die Menschenfresserei praktizierten. Der dissidente Geist dieser «aufmüpfigen Kulturtheorie» (Seefranz, 2013, S. 18) fand in der kulturkritischen Perspektive der Psychoanalyse und in deren Absicht, «das Unbewusste von den archaischen Strukturen zu befreien» (Plotkin, 2009, S. 73), viel Nahrung. Seefranz zeigt in ihrem Buch auf, wie eigenwillig die antropofagischen Modernisten sich den psychoanalytischen Diskurs aneigneten, wie sie das freudsche Gedankengut ihrem antropofagischen Einverleiben aussetzten.

Bindeglied zwischen der Psychoanalyse und dieser kulturellen Avantgarde war Durval Marcondes, ein ehemaliger Literaturlehrer und Schüler von Franco de Rocha, der sowohl in derWelt der Psychiatrie wie auch als wichtige und einflussreiche Figur in der kosmopolitischen Kulturszene São Paulos verkehrte. Er nahm die psychoanalytische Lehre von Franco de Rocha begierig auf und schrieb 1926 aus psychoanalytischer Sicht eine Arbeit über die ästhetische Symbolik in der Literatur. Er schickte sein Buch Sigmund Freud nach Wien. Ermutigt durch Freuds wohlwollende Antwort gründete Marcondes 1927 zusammen mit seinem Lehrer Franco de Rocha die erste psychoanalytische Gesellschaft Lateinamerikas, die "Sociedade Brasileira de Psicanálise”, welche eine provisorische Anerkennung durch die IPA erhielt. Um den geringen Einfluss auf die wenig psychoanalysefreundliche Welt der Psychiatrie São Paulos zu erhöhen, suchten sie eine Allianz mit Exponenten der Psychiatrie in Rio und fanden sie in Juliano Moreira und Júlio Pires Porto-Carrero. Bald entstand je eine Sektion in Rio und São Paulo. Während die Sektion in São Paulo ihre Akzeptanz in verschiedenen gesellschaftlichen Kreisen suchte, also v. a. auch unter Kulturschaffenden und Intellektuellen, engte sich die Sektion von Rio auf die Medizin ein. Damit erfolgte eine wichtige Weichenstellung für die Zukunft der brasilianischen Psychoanalyse.

1923 wurde in Rio de Janeiro die "Liga Brasileira de Higiene Mental" (Brasilianische Liga für geistige Gesundheit) gegründet, mit dem Ziel, die Versorgung psychisch Kranker zu verbessern. Ab 1926 wurde diese Institution immer mehr von der Ideologie der Eugenik durchdrungen (Nogueira do Vale, 2003). Für die brasilianische Elite war die Eugenik ein Symbol für Modernität, ganz im 
Sinne des Leitspruchs “Ordem e Progresso" (Ordnung und Fortschritt), welcher seit der Proklamation der Republik 1889 die brasilianische Flagge ziert. Zu den wichtigen Themen der Eugenik gehörten hygienische Erziehung, Psychohygiene, Sexualerziehung, Kontrolle von Eheschliessung und der Immigration, bis hin zu Überlegungen zur Sterilisation und Debatten über die Eindämmung der Rassenvermischung. Brasilien war das erste Land, in welchem eine «organisierte Bewegung zur Verbesserung der Volksgesundheit» entstand (Mendonça da Silva, 2011, S. 8). Das damalige Wirken der brasilianischen Medizin und Psychiatrie muss in diesem Kontext verstanden werden. Freuds Ideen kamen laut Montechi (2002) zu einer Zeit nach Brasilien, in welcher Themen wie Sexualerziehung, geistige Gesundheit und ganz allgemein Bevölkerungskontrolle unter hygienischen Gesichtspunkten für den gesellschaftlichen Fortschritt eine wichtige Bedeutung hatten. Júlio Pires Porto-Carrero, der zusammen mit Juliano Moreira 1928 die oben erwähnte 2. Sektion der "Sociedade Brasileira de Psicanálise" gründete, war ein wichtiger Vertreter der sich langsam etablierenden Psychoanalyse in psychiatrischen Kreisen von Rio de Janeiro. Er setzte sich - durchaus dem Geist der Eugenik verpflichtet - intensiv mit Erziehungsfragen und insbesondere auch mit der Sexualerziehung auseinander. Er wurde ein hartnäckiger Verfechter der Einführung psychoanalytischer Erkenntnisse in die Pädagogik und gründete im Schoss der "Liga Brasileira de Higiene Mental" 1926 die erste psychoanalytische Klinik Brasiliens. Diverse Mediziner publizierten in dieser Pionierzeit Arbeiten zu Freuds Sexualtheorie. In seiner Dissertation “Médicos, psicanalistas e loucos” («Mediziner, Psychoanalytiker und Verrückte») kommt Fidelis da Ponte zu folgendem Schluss:

Die Psychoanalyse war der Logik der Eugenik unterworfen, welche die Mediziner und Intellektuellen jener Zeit in Bann zog. Diese Unterordnung verzerrte diverse ihrer Vorstellungen und erschwerte ihre Verbreitung als eigenständige und von den Konzepten, welche in den degenerativen Prozessen die Ursachen für psychische Störungen sahen, radikal unterschiedliche Disziplin. (Fidelis da Ponte, 1999, S. 30; Übersetzung: G. B.)

Wenn die Mediziner die Psychoanalyse als «Werkzeug zum besseren Verständnis und zur Domestizierung der wilden Anteile der brasilianischen Kultur» (Plotkin, 2009, S. 72) benutzten, so sahen die Kulturschaffenden in der Psychoanalyse eine Methode, gerade diese wilden und exotischen Anteile ästhetisch 
zu überhöhen und als Metaphern zur Kritik an den herrschenden Verhältnissen zu benutzen.

Etwas zugespitzt könnte man sagen: Während sich die Psychoanalyse in Rio in den Anfängen eher im Geist einer dem Fortschrittsglauben verpflichteten Kontrolle derVolksgesundheit entwickelte, war der Nährboden für ihre Verbreitung in São Paulo die Aufbruchstimmung einer gesellschaftlichen Bewegung, welche Konventionen radikal in Frage stellte. Die Psychoanalyse stellte sowohl für die kontrollierend-strukturierende als auch für die aufbrechende Tendenz Denk- und Interpretationsmuster zur Verfügung.

Die Aufbruchstimmung durch die Gründung der ersten psychoanalytischen Gesellschaft Lateinamerikas, der “Sociedade Brasileira de Psicanálise”, 1927 kam bald ins Stocken. Die ein Jahr später gegründete Zeitschrift "Revista Brasileira de Psicanálise" in São Paulo überlebte mangels Interesse die Erstausgabe nicht, da die grosse Anhängerschaft unter den Kulturschaffenden keine berufliche Karriere als Psychoanalytiker anstrebten (Plotkin, 2009, S. 76). Nachdem die IPA beschlossen hatte, die am Berliner Psychoanalytischen Institut praktizierte dreigliedrige psychoanalytische Ausbildung als international verbindlichen Standard einzuführen, setzte sich Marcondes dafür ein, die Voraussetzungen für die Einführung dieses Systems in Brasilien zu schaffen. In der Folge löste sich die "Sociedade Brasileira de Psicanálise" 1930 auf.

Marcondes Suche nach europäischen Lehranalytikern gestaltete sich schwierig. Viele deutsche und österreichische Psychoanalytiker, die vor der Repression und der Verfolgung des aufkommenden Nationalsozialismus flohen, emigrierten in die USA. Erst 1936 gelang es, Adelheid Koch vom Berliner Psychoanalytischen Institut als erste Lehranalytikerin nach Brasilien zu holen. Nach der Pionierphase begann somit über die Möglichkeit zur psychoanalytischen Ausbildung der Prozess der Institutionalisierung der brasilianischen Psychoanalyse. In Rio sollte es bis zur Ankunft der ersten Lehranalytiker noch bis Ende der 40er-Jahre dauern. Wegen dieser schwierigen Rekrutierung von Lehranalytikern und infolge der Installierung eines autoritären Regimes durch Getúlio Vargas 1930, welches von 1937-45 als “Estado Novo” in eine Diktatur mündete, suchten nicht wenige angehende brasilianische Psychoanalytiker eine Ausbildung in der kleinianisch orientierten Psychoanalyse in Argentinien, welche sich dort mittlerweile formiert hatte. 1948 kamen aufVermittlung von Ernest Jones hin gleich zwei Lehranalytiker nach Rio: aus England Mark Burke und aus Deutschland Werner Kemper. Um beide herum formierten sich Gruppen, die in der Folge nicht zuletzt deshalb stark miteinander zu rivalisieren begannen, weil Kemper als ehemaliges Mitglied des 
Göring-Institutes und Burke als polnischer Jude aus völlig verschiedenen Lagern des kriegsgeschüttelten Europa kamen. Neben diesen zwei Gruppen formierte sich zur selben Zeit eine dritte, die «Argentinier», welche aus dem Exil zurückkehrten und dem kleinianischen Denken in Brasilien zu Einfluss verhalfen. 1945 erhielt die psychoanalytische Gruppe, welche sich um Adelheid Koch formierte, als “Sociedade Brasileira de Psicanálise do São Paulo” die provisorische Anerkennung und 1951 die definitive durch die IPA. 1955 wurde diese Anerkennung der Gruppen um Kemper als “Sociedade Psicanalítica de Rio de Janeiro” (SPRJ) zuteil und 1957 den zwei anderen Gruppen, die sich in der "Sociedade Brasileira de Psicanálise de Rio de Janeiro” (SBPRJ) zusammengeschlossen hatten.

\section{Brüche}

Figueiredo (2006) stellte in ihrem Aufsatz "Brilhante história da Psicanálise no Brasil” («Die schillernde Geschichte der Psychoanalyse in Brasilien») dar, wie sich die offizielle Psychoanalyse an den Universitäten und an den psychologischen Fakultäten ausbreitete, sich mit der klinischen Psychologie langsam verwob und mit der Zeit konfliktuös verstrickte. Auch am Anfang dieser Geschichte war Marcondes, der 1939 den ersten Lehrstuhl für Psychoanalyse an einer Universität erhielt, an der "Escola Livre de Sociologia e Política". Zu Beginn entwickelte sich die psychoanalytisch orientierte klinische Psychologie v. a. über die Kinderpsychoanalyse. 1962 wurde der Berufsstand der Psychologen reglementiert, was den Psychologen die Möglichkeit zur klinischen Praxis eröffnete. Mit einer Universitätsreform wurde 1968 die Privatisierung der Universitäten begünstigt, was zur Entstehung von vielen Angeboten an psychologischen Ausbildungsgängen führte. Dies bewirkte einen grossen Andrang an den psychologischen Fakultäten. Es entwickelte sich ein regelrechter "Psychoboom», der ab Ende der 60er und v. a. in den 70er-Jahren mit einer Popularisierung der Psychoanalyse über die Medien einher ging (Fidelis da Ponte, 1999, S. 63) und zum Phänomen der Warteschlangen vor den psychoanalytischen Praxen führte. Verstärkt wurde dieses Phänomen durch den wirtschaftlichen Aufschwung, den die Diktatur ab 1964 bewirkte. Die städtische Mittelschicht erhielt dadurch neue Konsummöglichkeiten, andererseits wurde durch die Repression die Meinungsfreiheit stranguliert. Die psychoanalytischen Praxen boten sich in dieser Situation als finanziell erschwingliche Rückzugsräume an. Die Psychologen begannen aber als Analysanden nicht nur einen fruchtbaren Markt für die ersten Generationen von Psychoanalytikern darzustellen, sondern sie wurden mit der Möglichkeit zur Berufsausübung auch zu deren Konkurrenten. Laut Chebabi (2000) begann die Aussicht auf eine finanziell lukrative Karriere durch den 
«Psychoboom» zu einem wichtigen Motiv bei der psychoanalytischen Ausbildung und der Berufsausübung zu werden. Zu Beginn besetzten die Psychologen auf dem umstrittenen Psychomarkt die Kinderpsychoanalyse, da «viele glaubten, die Behandlung von Kindern sei einfacher» (Figueiredo, 2006, S. 3; Übersetzung: G. B.). Langsam begannen die Psychologen aber die klinische Praxis in der Funktion von Therapeuten und gar als Supervisoren zu dominieren. Sie übernahmen auch wichtige Positionen im Lehrbetrieb als Vermittler der Psychoanalyse an neue Generationen von Psychologen und Psychoanalytikern. In den 70er-Jahren wurden immer mehr Gruppen gegründet, in denen Psychoanalytiker und klinische Psychologen gemeinsam Veranstaltungen organisierten, um das psychoanalytische Gedankengut zu verbreiten. Diese Gruppen funktionierten als Vermittler zwischen dem prestigeträchtigen Berufsstand der Psychoanalytiker und einer wachsenden Anzahl psychoanalytisch orientierter Psychologen. Das Verhältnis zwischen den zwei Berufsgruppen wurde aber zusehends problematischer, da es sich nicht um eine Auseinandersetzung zwischen zwei gleichberechtigten Partnern handelte. Die Zulassungskriterien zum offiziellen Berufsstand der Psychoanalytiker waren rigide, v. a. in Rio de Janeiro, wo in den offiziell von der IPA anerkannten Psychoanalytischen Gesellschaften der Zugang für Laienanalytiker versperrt war, d.h. ausschliesslich Mediziner zugelassen wurden.

In einem Journal-Artikel von 2004 mit dem Titel «Les États Généraux de la Psychanalyse in Rio de Janeiro und die brasilianische Psychoanalyse» (Busslinger, 2004) habe ich beschrieben, wie die offiziellen Psychoanalytischen Gesellschaften im Verlauf der 70er-Jahre zusehends ins Kreuzfeuer der Kritik gerieten, nicht nur, weil ihre rigiden Zulassungskriterien infolge der sich ausbreitenden psychoanalytischen Berufspraxis immer mehr auf Widerstand stiessen, sondern auch weil sich 1973 ein Skandal um die Involvierung von Psychoanalytikern in Folter und Repression des Militärapparates ereignete. Obwohl es der offiziellen brasilianischen Psychoanalyse mit Hilfe der IPA über Ausschluss und Zensur gelang, den Skandal vorerst zu vertuschen, traten die Brüche im psychoanalytischen Gebäude immer mehr zutage. Chnaiderman (1998) beschreibt, wie schon in den 50er- und 60erJahren vereinzelt psychoanalytische Gesellschaften gegründet wurden, die nicht der IPA angehörten und wie in den 70er-Jahren - nicht zuletzt infolge des vertuschten Folterskandals - verschiedenste psychoanalytische Gruppierungen aus dem Boden schossen, die sich allesamt von den IPA-Organisationen abgrenzten und in denen Laienanalytiker einen Platz fanden. Die Vorwürfe dieser Gruppierungen konzentrierten sich auf die erstarrte Hierarchie in den IPA-Gesellschaften. Von einer «Gerontokratie» war die Rede (Füchtner, 2002, S. 77), in welcher der Status der 
Lehranalytiker wie Pfründe verteilt und auf Lebzeiten gehortet würden. Das Klima innerhalb der IPA-Gesellschaften wurde als ein «feudales» kritisiert (Busslinger, 2004). Laut Chebabi (2000) erhielt die Frage nach den Kriterien der psychoanalytischen Ausbildung durch die Verteilungskämpfe, die sich mit dem Abflauen des «Psychobooms» in den 90er-Jahren zuspitzten, eine zusätzliche Brisanz. Die ärztlichen Psychoanalytiker versuchten in diesem Kampf vergeblich, ihr Monopol gegenüber den Laienanalytikern per Gesetz zu verankern (Fidelis da Ponte, 1999, S. 88).

\section{Entfaltung im Auseinanderbrechen}

Es würde den Rahmen dieses Artikels sprengen, auf die unterschiedlichen theoretischen, organisatorischen und berufspolitischen Ausrichtungen all der Institute einzugehen, die durch die Krise der offiziellen Psychoanalyse entstanden sind. An dieser Stelle möchte ich lediglich auf zwei Phänomene eingehen, welche dazu beitrugen, die psychoanalytische Landschaft in der Folge dieser Krise zu verändern und zu diversifizieren.

Die Krise spitzte sich in einem Moment $\mathrm{zu}$, als viele argentinische Psychoanalytiker infolge der zunehmenden Repression der argentinischen Militärdiktatur ab Mitte der 70er-Jahre nach Brasilien auswanderten, da die brasilianische Diktatur weniger repressiv war. Viele dieser Exilanten vertraten eine unorthodoxe Sichtweise der Psychoanalyse. Sie verstärkten die Tendenzen des sozialen Engagements der Psychoanalyse mit den weniger privilegierten sozialen Schichten, welche durch Gruppen wie z. B. die 1973 gegründete “Clínica Social de Psicanálise” schon existierten. Sie führten neue Praktiken der psychoanalytischen Arbeit mit Gruppen ein und trugen zur Verstärkung der Kritik an der «pseudowissenschaftlichen Neutralität» der offiziellen Psychoanalyse bei (Fidelis da Ponte, 1999, S. 73). In den 70er-Jahren wurden die ersten lacanschen Institute gegründet. Für viele Psychologen, die einen psychoanalytischen Werdegang anstrebten, wurde die lacansche Psychoanalyse eine interessante Alternative hinsichtlich des umstrittenen Zugangs zum Status eines Psychoanalytikers - und zwar sowohl in berufspolitischen Belangen wie auch bezüglich theoretischer Fragen (Figueiredo, 2006). Der Ausschluss von Lacan aus der IPA 1963 führte zu einer institutionskritischen Neuformulierung der Ausbildungskriterien von Seiten der lacanschen Schulen. Insbesondere die Bürokratisierung und der rigide Umgang mit der Lehranalyse wurde kritisiert und es wurden neue Zugangskriterien zum Status des Psychoanalytikers definiert. Diese kritische Position eröffnete den Psychologen mit universitärem Werdegang den von den orthodoxen Instituten verwehrten 
Zugang zum begehrten Status eines Psychoanalytikers. Füchtner schrieb in diesem Zusammenhang in seinem Aufsatz «Wuchernde Psychoanalyse. Die brasilianische Psychoanalyse in der Krise» von «formal relativ unpräzisen und im Vergleich zu den IPA-Gesellschaften weniger strengen Ausbildungsvorschriften», die einen breiteren und offeneren Zugang zur psychoanalytischen Ausbildung ermöglichten (Füchtner, 2002, S. 74).

Aber nicht nur diese berufspolitische Öffnung machte ein Studium der lacanschen Psychoanalyse attraktiv, sondern auch das Motto «Zurück zu Freud» unter dem Lacan seine Theorie formulierte. Die Entwicklung der Öffnung der Psychoanalyse für die Psychologen über die universitäre Vermittlung seit den 50erJahren wurde von gewissen Sektoren der IPA-Gesellschaften mit kritischen Augen verfolgt. So kommentierte z. B. Leão Cabernite, der Präsident einer der zwei grossen IPA-Gesellschaften Rios, der als Lehranalytiker des beschuldigten Arztes direkt in den Folterskandal der 70er-Jahre verwickelt war, diese Entwicklung ziemlich polemisch folgendermassen:

Eine lärmende und wachsende Legion von Psychopathen hat die Psychoanalyse gestürmt. Leute mit Persönlichkeitsstörungen beflügeln sich, Psychoanalytiker zu sein. Nach und nach wird die zerstörerische Invasion des Berufsstandes in eine alarmierende Zerstörung der Psychoanalyse als Wissenschaft münden. (Fidelis da Ponte,1999, S. 74; Übersetzung: G. B.)

Auch wenn solche Aussagen kaum repräsentativ sind, so wurden sie doch von einem Exponenten der offiziellen Psychoanalyse gemacht und zeigen etwas von der Dominanz der Definitionsmacht der Mediziner auf.

Die lacansche Theorie stellte nun mit ihrer strukturalistischen, philosophischen und kulturkritischen Neuausrichtung einen Gegenpol zur Haltung dar, welche durch solche Aussagen zum Ausdruck kam und hatte eine legitimierende Wirkung für psychoanalytisch orientierte Universitätspsychologen. Es hat wohl auch mit einer relativ unverbindlichen Struktur und Organisation zu tun, dass die Szene der lacanschen Schulen und Gruppen in Brasilien mit der Zeit unübersichtlich geworden ist. «Der Lacanismus ist heterogen als Bewegung und es mangelt nicht an endlosen Diskussionen über die unterschiedlichen Lektüren des Meisters» (Figueiredo, 2006, S. 12; Übersetzung: G. B.). Füchtner schätzt, dass von den etwa 40 psychoanalytischen Gruppen in Rio de Janeiro anfangs der 90er-Jahre etwa die Hälfte lacanianisch orientiert war (Füchtner, 2002, S. 73). 
Zum Schluss möchte ich auf die Frage nach der Spezifik der brasilianischen Psychoanalyse eingehen. Sicher nicht spezifisch ist das Abflauen des Booms der Psychoanalyse anfangs der 90er-Jahre zugunsten der Neurowissenschaften und Therapiemethoden, wie derVerhaltenstherapie, welche den Kriterien von Effizienz und Kontrollierbarkeit der beginnenden Globalisierungstendenzen scheinbar besser entsprachen.

In der Diskussion um die Spezifik der brasilianischen Psychoanalyse taucht in der Fachliteratur immer wieder der Einfluss des antropofagischen Diskurses des Modernismus auf. Wie ich oben aufzuzeigen versuchte, hat sich dadurch in São Paulo eine weniger medizinalisierte Rezeption der Psychoanalyse durchgesetzt als in Rio de Janeiro. Nichtsdestotrotz war und blieb das Mekka der brasilianischen Psychoanalyse in Europa, erst in London und seit der Etablierung der lacanschen Schule in Paris (Chnaiderman, 1998). Andererseits hat die Kompromittierung der offiziellen brasilianischen Psychoanalyse während der Diktatur nicht nur - wie oben beschrieben - zu einer lebendigen und facettenreichen psychoanalytischen Landschaft in Brasilien beigetragen, sondern weil die IPA bei der Vertuschung des Folterskandals Schützenhilfe geleistet hatte (Busslinger, 2004, S. 34), strahlte dieser Skandal auch international aus. René Major, der sich schon länger für eine institutionskritische Haltung einsetzte - u. a. auch über die Vernetzungsbewegung der 70er-Jahre, in welcher das Psychoanalytische Seminar Zürich eine wichtige Rolle spielte -, trat aus Protest gegen diese Schützenhilfe 1996 aus der IPA aus und rief die «États Generaux de la Psychanalyse» (EGP) als Gegenbewegung zur Haltung der offiziellen Psychoanalyse ins Leben. Auf seine Initiative hin fand nach dreijähriger Vorbereitung durch 160 Psychoanalytiker der erste Kongress im Juli 2000 in Paris statt. Um die tausend TeilnehmerInnen aus mehr als 30 Ländern und aus verschiedenen psychoanalytischen Schulen setzten sich aus einer institutionskritischen Haltung heraus über die Krise und die Zukunft der Psychoanalyse auseinander (siehe Journal für Psychoanalyse 2004, Nr. 43, welches unter dem Titel «Vernetzung der freudschen Linken» dieser Bewegung einige Artikel widmete). Wenn die Auseinandersetzungen in Brasilien rings um die sich abschottenden IPA-Gesellschaften ein wichtiger Antrieb für die Entstehung der EGP waren, so war die EGP andererseits ein bedeutsames Vehikel für eine stärkere Auseinandersetzung zwischen den verschiedenen psychoanalytischen Gruppen, Institutionen und Schulen in Brasilien. Entsprechend war die Präsenz der brasilianischen Psychoanalyse am Kongress in Paris gross. Laut Michel Sauval kamen mit mehr als 200 Teilnehmern noch nie derart viele Vertreter der brasilianischen Psychoanalyse ausserhalb des Landes zusammen. Mit 35 Prozent der Beiträge 
belebte die brasilianische Präsenz die Auseinandersetzung wesentlich (Guillermo, 2002, S. 6). 2003 fand der zweite Kongress von "Estados Gerais da Psicanálise" (portugiesische Übersetzung von «États Generaux de la Psychanalyse») in Rio de Janeiro statt. Insgesamt vier Vorbereitungstreffen mit Beteiligung von Vertretern verschiedener psychoanalytischer Gruppen und Institutionen (auch der IPAGesellschaften), die «alle im eigenen Namen sprachen, ohne sich hinter der Haltung einer Institution zu verstecken» (so das Motto von EGP), trugen zu einer lebendigen Auseinandersetzung innerhalb der brasilianischen Psychoanalyse bei.

Diese Beteiligung der brasilianischen Psychoanalyse an einer international abgestützten institutionskritischen Bewegung beantwortet die Frage nach der Spezifik der brasilianischen Psychoanalyse allerdings kaum. Es ist eine Frage, die wohl auch nur spekulativ beantwortet werden kann. In diesem Sinne lasse ich zum Schluss einen Vertreter der institutionskritischen Psychoanalyse zu Wort kommen. Joel Birman, einer der Organisatoren von EGP in Rio de Janeiro, der die Zukunft der Psychoanalyse mehr in psychoanalytischen Arbeitsgruppen als in den etablierten psychoanalytischen Institutionen sieht, skizziert 2012 in einem Interview folgendes Bild der aktuellen brasilianischen Psychoanalyse:

Unsere Schwierigkeit besteht darin, einen eigenen psychoanalytischen Diskurs zu etablieren, unseren Erfahrungen, unseren Ambitionen, unseren Wünschen einen Wert zu geben und das Beste aus der internationalen psychoanalytischen Tradition herauszufiltern, denn die Zeiten der nationalen Diskurse sind vorbei. Wir müssen das Beste, das sich uns anbietet, einverleiben und derart unserer Erfahrung ein Gewicht und einen Wert geben. Meiner Ansicht nach wurde die brasilianische psychoanalytische Tradition infolge einer kolonialistischen Mentalität beeinträchtigt. Sie hat es verpasst, dieser Einverleibung durch eine konzeptionelle Verarbeitung von Freud einen Wert zu geben, handle es sich nun um die englische Schule, die Amerikaner oder um eigene Vorstellungen, die sehr durch kolonialistische Einflüsse bestimmt waren. Das heisst, man ist mit einer verblüffenden Leichtigkeit von der englischen zur französischen Schule übergegangen, von Melanie Klein zu Lacan und man behandelt Lacan, wie man Melanie Klein behandelt hatte, mit der gleichen Art von Glaube, den ich in den 60er Jahren erlebt habe. Die Leute sprachen von sehr komplizierten Dingen wie der paranoid-schizoiden und der depressiven Position und die Leute 
beziehen sich heute auf Lacans "reinen Signifikanten», auf das Reale, wie wenn es sich um äusserst komplizierte Banalitäten handeln würde, aber mit der gleichen Art von Glauben. (...) eine Mittelmässigkeit, die aus einem Mangel an Neugierde entsteht, einem Mangel an Wertschätzung gegenüber dem nationalen Erfahrungsschatz, währendem jeder Franzose uns Brasilianer z. B. für unseren kreativen Umgang mit der Klinik bewundert, genau deshalb weil wir es ganz anders machen. Unserem "scheiss verrückten" Potenzial gegenüber haben sie jene halbwegs Angst einflössende normative Geschichte usw., was bedeutet, dass wir eine Kreativität haben, welche sie entbehren, nicht weil sie schlechter sind als wir, sondern weil wir weniger belastet sind durch 300 oder 400 Jahre cartesianische Tradition. Nun gut, wir sind deshalb nicht besser, aber dies gibt uns eine Freiheit, die sie nicht haben. (Birman, 2012, S. 4 f.; Übersetzung: G. B.)

\section{Literatur}

Birman, Joel. (2012). O futuro da psicanálise não está nas grandes instituições. Revista Revide. http://www.revide.com.br/blog/luis-fernando-s-de-souzapinto/ o-futuro-da-psicanalise-nao-esta-nas-grandes-insti/.

Busslinger, Gregor. (2004). Les États Généraux de la Psychanalyse in Rio de Janeiro und die brasilianische Psychoanalyse. Journal für Psychoanalyse, 43, 31-44.

Chebabi, Wilson de Lyra. (2000). Crônica de uma controvérsia crônica. http://egp. dreamhosters.com/textos/chebabi_wilson_de_liracronica_de_uma_controversia.shtml.

Chnaiderman, Miriam. (1998): Existe uma Psicanálise Brasileira? Percurso Nr. 20, São Paulo: Departamento de Psicanálise do Instituto Sedes Sapientiae.

Fallend, Karl. (2010). «Carusos Erben». Reflexionen in einer erhitzten Auseinandersetzung. Werkblatt 64. Zeitschrift für Psychoanalyse und Gesellschaftskritik, 100-127.

Fidelis da Ponte, Carlis. (1999). "Médicos, psicanalistas e loucos: uma contribuição à história da psicanálise no Brasil”. Mestrado na Fundação Oswaldo Cruz, Escola Nacional de Saúde Pública.

Figueiredo Ana Cristina. (2006). Brilhante história da Psicanálise no Brasil. Rio de Janeiro: Antroposmoderno. 
Füchtner, Hans. (2002). Wuchernde Psychoanalyse. Die brasilianische Psychoanalyse in der Krise. Werkblatt 49. Zeitschrift für Psychoanalyse und Gesellschaftskritik, 63-87.

Galdini Raimundo Oda, Ana Maria. (2001). História da Psiquiatria. A teoria da degenerescência na fundação da psiquiatria brasileira. Contraposição entre Raimundo Nina Rodrigues e Juliano Moreira. Psychiatry on line Brasil, Vol. 6, Nr. 12.

Kemper, Jochen. (1988). Brief von Jochen Kemper an die Psychoanalytische Gesellschaft von Rio de Janeiro. Psyche 1988/11, 1016-1020.

Mendonça da Silva, Henrique. (2011). A assistência médico-social como campo eugênico na obra do médico Luiz Palmier. Anais do XXVI Simpósio Nacional da História - ANPUH, São Paulo.

Mokrejs, Elisabete. (1992). A Psicanálise no Brasil. Petrópolis (Vozes).

Montechi Valladares de Oliveira, Carmen Lucia (2002). Os primerios tempos da psicanálise no Brasil e as teses pansexualistas na educação, Ágora: Estudos em Teoria Psicanalítica v.V n. 1, Rio de Janeiro, 133-154.

Nogueria do Vale, Eliana Araújo. (2003). Os rumos da Psicanálise no Brasil. São Paulo: Escuta.

Pietra, Guillermo. (2002). Reportaje a María Cristina Rios Magalhães. Acheronta, Revista de Psicoanálisis y Cultura 2002/16. www.acheronta.org.

Plotkin, Mariano Ben. (2009). Psicoanálisis y habitus nacional: un enfoque comparativo de la recepción del psicoanálisis en Argentina y Brasil (1910-1950). Memoria y sociedad, 27, 61-85, Bogotá.

Roudinesco, Elisabeth \& Plon, Michel. (2004). Wörterbuch der Psychoanalyse. Wien, New York: Springer.

Schneider, Galina. (1985). Stellungnahme zu traurige Psychotropen. Psyche 1985/12, 1133-1149.

Seefranz, Catrin. (2013). Tupi Talking Cure. Zur Aneignung Freuds im antropofagischen Modernismus Brasiliens. Berlin, Zürich, Wien: LIT. 